\title{
Cotinine concentrations in follicular fluid as a measure of secondhand tobacco smoke exposure in women undergoing in vitro fertilization: Inter- matrix comparisons with urine and temporal variability
}

\section{Citation}

Benedict, Merle D., Stacey A. Missmer, Allison F. Vitonis, Daniel W. Cramer, and John D. Meeker. 2011. "Cotinine Concentrations in Follicular Fluid as a Measure of Secondhand Tobacco Smoke Exposure in Women Undergoing in Vitro Fertilization: Inter-Matrix Comparisons with Urine and Temporal Variability." Chemosphere 84 (1) (June): 110-116. doi:10.1016/ j.chemosphere.2011.02.037.

\section{Published Version}

doi:10.1016/j.chemosphere.2011.02.037

\section{Permanent link}

http://nrs.harvard.edu/urn-3:HUL.InstRepos:27336534

\section{Terms of Use}

This article was downloaded from Harvard University's DASH repository, and is made available under the terms and conditions applicable to Other Posted Material, as set forth at http:// nrs.harvard.edu/urn-3:HUL.InstRepos:dash.current.terms-of-use\#LAA

\section{Share Your Story}

The Harvard community has made this article openly available.

Please share how this access benefits you. Submit a story.

\section{Accessibility}




\title{
Cotinine Concentrations in Follicular Fluid as a Measure of Secondhand Tobacco Smoke Exposure in Women Undergoing In Vitro Fertilization: Inter-matrix Comparisons with Urine and Temporal Variability
}

\author{
Merle D. Benedict ${ }^{1}$, Stacey A. Missmer ${ }^{2,3,4}$, Allison F. Vitonis ${ }^{3}$, Daniel W. Cramer ${ }^{3}$, and \\ John D. Meeker ${ }^{1}$ \\ ${ }^{1}$ Department of Environmental Health Sciences, University of Michigan, Ann Arbor, MI, USA \\ 2 Department of Epidemiology, Harvard School of Public Health, Boston, MA, USA \\ ${ }^{3}$ Department of Obstetrics, Gynecology and Reproductive Biology, Brigham and Women's \\ Hospital and Harvard Medical School, Boston, MA, USA \\ ${ }^{4}$ Channing Laboratory, Department of Medicine, Brigham and Women's Hospital and Harvard \\ Medical School, Boston, MA, USA
}

\begin{abstract}
We examined the relationship between cotinine measures in follicular fluid (FF) and urine to inform our exposure assessment strategy for an ongoing epidemiological study of secondhand tobacco smoke (STS) exposure and early pregnancy loss. Among subjects undergoing in vitro fertilization (IVF), we compared cotinine levels in paired urine and FF samples from the same women and examined FF cotinine levels over time. We found a weak rank-order relationship (Spearman $\mathrm{r}<0.2$ ) and poor agreement for classifying nonsmoking individuals as exposed to STS (sensitivity $=0.29-0.71$; specificity $=0.35-0.72$ ) between cotinine concentrations in FF and urine. We observed fair reliability (ICC $=0.42-0.52$ ) in FF cotinine concentrations from women undergoing multiple IVF cycles. If available, FF cotinine concentrations may be desired as a biomarker of low-level tobacco smoke exposure over urinary cotinine in studies of early reproduction. Collection of multiple FF samples for cotinine analysis may be needed to accurately represent long-term STS exposure.
\end{abstract}

\section{Key terms}

cotinine; follicular fluid; environmental tobacco smoke; in vitro fertilization; percent agreement; intraclass correlation coefficient

\footnotetext{
(C) 2011 Elsevier Ltd. All rights reserved.

Address correspondence to: John Meeker, ScD, Department of Environmental Health Sciences, University of Michigan School of Public Health, 6635 SPH Tower, 109 S. Observatory St., Ann Arbor, MI 48109, Phone: 1-734-764-7184, Fax: 1-734-763-8095, meekerj@umich.edu.

DECLARATION OF INTEREST

The authors report no declarations of interest.

Publisher's Disclaimer: This is a PDF file of an unedited manuscript that has been accepted for publication. As a service to our customers we are providing this early version of the manuscript. The manuscript will undergo copyediting, typesetting, and review of the resulting proof before it is published in its final citable form. Please note that during the production process errors may be discovered which could affect the content, and all legal disclaimers that apply to the journal pertain.
} 


\section{INTRODUCTION}

Active smoking is associated with adverse effects on fertility and early pregnancy (Cooper and Moley, 2008). Recent studies have also suggested that secondhand tobacco smoke (STS) exposure has deleterious effects on early reproduction (Neal et al., 2005; Peppone et al., 2009), though these studies rely on self-reported exposure. Conversely, an earlier study using a biomarker of tobacco smoke exposure found no difference in fertilization or pregnancy rates between active, passive and nonsmokers (Sterzik et al., 1996).

It is currently unclear which method of STS exposure assessment is most appropriate in studies of early pregnancy. Self-reported exposure is commonly used due to its convenience and economy, but is subject to recall bias and misclassification errors (George et al., 2006). Objective markers of tobacco smoke exposure, such as nicotine and its metabolites, are also frequently measured in biological samples. Approximately $75 \%$ of nicotine is converted to cotinine in humans. Cotinine is a widely accepted biomarker because of its specificity and relatively long half-life in body fluids ( 16 hours) compared to nicotine ( $\sim 2$ hours; Benowitz et al., 2009a). However, a single measure of cotinine in fluids such as blood or urine may be limited because it reflects only recent exposure (3-4 days; Benowitz et al., 2009a; Matt $e t$ al., 2007).

Assisted reproduction technologies (ART) provide the opportunity to study the effects of environmental insults on stages of early reproduction otherwise not observable (e.g. fertilization, implantation). ART also facilitate the collection of follicular fluid (FF), which surrounds the preovulatory oocyte. FF is composed of constituents of blood plasma that cross the blood-follicle barrier as well as secretions from granulosa and thecal cells (Fortune, 1994). FF provides a very important microenvironment in which the oocyte matures and granulosa cells differentiate (Fahiminiya and Gerard, 2010; Fortune et al., 2004).

Since the ovarian follicle has no direct blood supply, in order for cotinine and other chemicals to enter FF they must diffuse through interstitial fluid and/or be transported through thecal and granulosa cells which surround the antrum and oocyte (Fabro, 1978). Gap junctions (nonspecific pores between cells) can transport molecules up to $1 \mathrm{kDa}$ in molecular mass and, since cotinine has a molecular mass of only 176.2 Da, are likely involved in the passive transport of cotinine into FF (Weber et al., 2004). Once within the $\mathrm{FF}$, cotinine and other chemicals can interact directly with the cumulus-oocyte complex and may contribute to adverse effects on fertilization and blastocyst implantation (Fabro, 1978). Thus, cotinine measured in FF, as opposed to other media (e.g. urine, serum), may be a more appropriate biomarker of tobacco smoke exposure in studies of early pregnancy because it reflects a maturing oocyte's direct exposure to the constituents of tobacco smoke.

Cotinine was first measured in FF in smokers in 1989 (Weiss and Eckert, 1989), and in 1996, Zenzes et al. detected cotinine in the FF of passive smokers (i.e. those exposed to STS). Shortly thereafter, Zenzes and Reed (1998) demonstrated the ability of FF cotinine to discriminate between active, passive and nonsmokers.

Using urinary cotinine concentrations to estimate exposure in a large cohort of women undergoing in vitro fertilization (IVF), we recently reported no increased risk of failed implantation or spontaneous abortion among exposed nonsmokers compared to those unexposed (Meeker et al., 2007). However, few studies have examined whether urinary cotinine is strongly related to more biologically relevant markers of STS exposure in studies of early pregnancy (e.g. cotinine in FF). Cotinine concentrations in urine are measures of plasma cotinine not reabsorbed during glomerular filtration; while cotinine concentrations in FF represent plasma cotinine that diffused through the blood-follicle barrier and largely 
depend upon concentration gradients between the blood, interstitial fluid, and cells surrounding the oocyte. In addition to these biological differences, relying on biomarkers more remote to the target area (e.g. gametes) may increase exposure measurement error and misclassification, which tend to bias effect estimates toward the null (Armstrong, 1998). Thus, we hypothesized poor agreement between cotinine concentrations in follicular fluid and urine, which may partially explain the lack of association between exposure and pregnancy outcomes in our previous report.

In the present study we compared cotinine levels in paired urine and FF samples from the same women. We also examined FF cotinine levels over time among subjects undergoing multiple IVF treatment cycles to determine if repeated exposure measures would be needed in epidemiology studies of STS exposure and IVF outcomes. These efforts were intended to inform our exposure assessment strategy for a large, ongoing epidemiological study of STS exposure and early pregnancy loss among ART patients. As far as we are aware, this is the first study to examine the relationship between cotinine concentrations in FF and urine and the intra-individual variability of FF cotinine concentrations over time.

\section{METHODS}

\subsection{Study Population}

For the present study, 415 subjects were randomly selected from among those who underwent oocyte retrieval (i.e. for whom a FF sample was taken) in a larger study previously described (Meeker et al., 2007). Briefly, in the larger study, couples undergoing IVF or intracytoplasmic sperm injection (ICSI) between 1994 and 2003 were recruited through three Boston-area clinics to study predictors of IVF success. Protocols were approved by the Human Research Committees at Brigham and Women's Hospital, the Harvard School of Public Health, and the University of Michigan. Approximately $65 \%$ of couples approached agreed to participate in the study. Couples excluded from the study were those who underwent gamete intra-fallopian transfer (GIFT) or were gestational carriers, as well as those who required donor oocytes or donor semen. After exclusions, 2,350 couples who underwent from one to six IVF/ICSI treatment cycles were enrolled in the parent study.

\subsection{Exposure Assessment}

A self-administered questionnaire was used to obtain information from each subject on medical history and lifestyle factors such as: demographics, ages of both male and female partner, medical and reproductive history, smoking history, and duration of infertility. Selfreported STS exposure at home or at work was also obtained as well as information on male partner's present smoking status.

Physicians and technicians were asked to retain the FF from study patients during egg retrieval for each cycle. FF was aspirated from follicles using a 16-gauge needle and constant suction from a Rocket pump apparatus. Fluid was collected from the largest visible follicle before using any flushing medium and then transferred to a sterile Petri dish. Oocytes were scanned for and removed. The fluid, normally discarded at this point, was placed into a $15 \mathrm{~mL}$ conical tube and centrifuged for 15 minutes. The supernatant was placed into a clean storage tube, labeled, refrigerated, and transferred to the Brigham and Women's Hospital laboratory within 12 hours. At the laboratory, the specimens were aliquoted into $2 \mathrm{~mL}$ specimens and frozen at -80 degrees centigrade. FF was analyzed for cotinine using a quantitative enzyme-linked immunosorbent assay (ELISA; BioQuant, Inc., San Diego, CA). This single-step, competitive test uses spectrometric measurement to determine cotinine in body fluids. It has a lower reporting limit of $0.3 \mathrm{ng} / \mathrm{mL}$ and inter- and intra-assay variations of 4 and $6 \%$, respectively. 
A first-morning, void urine sample was collected from participants in only the first half of the parent study (1994-1998). These samples were available for 255 of the 415 subjects in the present sub-study. Urine samples were collected in a sterile, wide-mouthed, 1-liter plastic container, aliquoted in the laboratory, and frozen at -80 degrees centigrade. Samples were later analyzed for cotinine via competitive radioimmunoassay techniques described elsewhere (Langone et al., 1973, Van Vunakis et al., 1993). The procedure had a lower reporting limit of $0.1 \mathrm{ng} / \mathrm{mL}$ and inter- and intra-assay variations of $5 \%$. After analysis, urinary cotinine concentrations were adjusted by creatinine $(\mathrm{Cr})$ to account for individual differences in hydration and urine output (Boeniger et al., 1993). Before we compared FF and urinary cotinine concentrations, we matched each subject's urine sample to the IVF/ ICSI treatment cycle that occurred nearest in time to its collection. Urine samples were matched to the initial cycle for $97 \%$ of the subjects in the present study.

\subsection{Statistical analysis}

Data analysis was performed using SAS software (version 9.2; SAS Institute Inc., Cary, NC). Quantified cotinine concentrations below the limit of detection (LOD) were kept as the reported value. Unquantified concentrations were assigned a value of one half of the LOD. We compared cotinine levels in urine and FF among the 255 subjects from whom both types of samples were collected. In preliminary analyses we tabulated cotinine distributions in both FF and urine, stratified by self-reported smoking status. Self-reported active smokers (n $=13$ ) were excluded to calculate Spearman correlation coefficients between concentrations of cotinine measured in paired FF and urine samples. We also conducted this correlation analysis after excluding smokers based on a published cutpoint or threshold (Fuentes et al., 2010). To determine whether the time between urine and FF sample collection affected the strength of cotinine correlation, we examined coefficients after stratifying subjects by the duration between sample collections.

We assessed the ability of a single urinary cotinine concentration to correctly classify an individual's STS exposure status by calculating its percent agreement with the individual's exposure classification based on the cotinine concentration in a paired FF sample. For this analysis we considered FF cotinine to represent a woman's "true" or "gold standard" exposure because it reflects a developing oocyte's direct exposure to the constituents of tobacco smoke. Also, FF samples were collected during IVF treatment at oocyte retrieval, while urine samples were often collected before oocyte retrieval. Thus, in studies of female fertility and early pregnancy, we hypothesize that FF cotinine represents a more biologically and, in our case, temporally relevant biomarker of STS exposure.

Among self-reported nonsmoking subjects in this agreement analysis, we considered those in the highest cotinine tertile as exposed. For comparison, we also used a published cutpoint to classify exposure. Following Zielinska-Danch et al. (2007), we categorized unexposed nonsmokers as those below an unadjusted urinary cotinine concentration of $50 \mathrm{ng} / \mathrm{mL}$. To the best of our knowledge, no STS exposure cutpoints exist for cotinine in Cr-adjusted urine or FF. Thus, we extended $50 \mathrm{ng} / \mathrm{mL}$ in unadjusted urine to our data distributions to establish cutpoints for cotinine in Cr-adjusted urine and in FF. We first determined the percentile in our unadjusted urinary cotinine distribution that corresponded to $50 \mathrm{ng} / \mathrm{mL}$, which was the $42^{\text {nd }}$ percentile. We then matched this percent rank to the $42^{\text {nd }}$ percent values in our FF cotinine and our Cr-adjusted urinary cotinine distributions, which were $1.11 \mathrm{ng}$ cotinine $/ \mathrm{mL}$ FF and 66 ng cotinine/g creatinine, respectively. These values were also used as STS exposure cutpoints for subsequent analyses. After nonsmoking participants were categorized as exposed or unexposed based cotinine concentrations in each sample media, sensitivity, specificity, and positive and negative predictive values were calculated and compared between classification methods (i.e. when using highest tertile or published cutpoints). 
Percent agreement was also assessed after stratifying cotinine concentrations by time between urine and FF sample collections.

Since women in the parent study underwent up to six IVF/ICSI cycles, we also examined the temporal variability (i.e. changes over time) in FF cotinine concentrations to assess how well a single FF cotinine measure may represent one's average exposure to STS over longer periods of time. Among all 415 subjects selected for the present study, we stratified FF cotinine levels by study year and examined cotinine distributions over time.

To assess the between- and within-person variability in FF cotinine concentrations, we calculated the intraclass correlation coefficient (ICC) and 95\% confidence intervals for lntransformed FF cotinine concentrations using SAS PROC MIXED (Hankinson et al., 1995). ICC is a measure of the reliability of repeat measures over time and is defined as the ratio of between-subject variance to total variance. Values range from zero to one indicating poor and excellent reliability, respectively (Rosner, 2000). The ICC was calculated for differing smoking groups for comparison (e.g. when including or excluding active smokers).

ICC is an indicator of reliability for continuous measures, though it does not quantify the degree of misclassification that may occur as subjects are categorized into exposure groups. Thus, we calculated the percent agreement of exposure categorization from a single cotinine measure to the category in which women were assigned when considering all their repeated FF cotinine measures (Mahalingaiah et al., 2008; Meeker et al., 2005). The geometric mean FF cotinine concentration for each subject was defined as their "true" exposure. As described earlier, a concentration of $1.11 \mathrm{ng}$ cotinine/mL FF was used to categorize women as exposed or unexposed to STS. Thus, we determined the percent agreement between each cycle and the subject's "true" exposure. Sensitivity, specificity, and positive and negative predictive values were evaluated and compared between smoking groups.

\section{RESULTS}

The 415 subjects selected for the present study had FF collected from one to six IVF/ICSI treatment cycles, with a mean of 1.8 cycles per women. The number of women who underwent 1, 2, 3, 4, 5, and 6 cycles, were, respectively, 209, 109, 63, 24, 7, and 3. A single urine sample was available for 255 subjects recruited between 1994 and 1998 (i.e. in the first half of the parent study). Detectable cotinine concentrations were measured in $100 \%$ of urine samples and $82 \%$ of FF samples.

Large differences $(\mathrm{p}<0.0001)$ were observed between the cotinine distributions of selfreported active smokers and nonsmokers and are presented in Table 1. No significant differences were found between the cotinine distributions of nonsmokers who reported they were exposed to STS and nonsmokers who did not report STS exposure. Cotinine concentrations were consistently higher in urine than in FF.

No clear relationship between urinary and FF cotinine concentrations was observed visually (Figure 1). Several self-reported nonsmokers had very high levels of cotinine in either FF or urine or both, which may reflect active smokers who inaccurately reported their smoking status. Overall, a weak correlation (Spearman $r<0.2$ ) was observed between cotinine concentrations in these two media and is presented in Table 2. Because of the short biological half-life of cotinine in body fluids, we hypothesized that the correlation between cotinine in urine and FF would strengthen as the time between collections of the two samples decreased. For subjects whose urine and FF samples were collected more than 15 days apart, correlation coefficients declined somewhat compared to all samples, as expected. Likewise, correlations were slightly strengthened among samples collected within 15 days of each other compared to all samples. 
However, as subjects were grouped by shorter time intervals between sample collections, we observed a decreasing trend in the strength of cotinine correlation. When a published cutpoint was used to distinguish active from passive smokers, coefficients decreased compared to coefficients calculated among self-reported nonsmokers. This is may be due to the presence of active smokers who misreported their smoking status. Thus, the lower half of the table is likely representing the true correlation of cotinine concentrations in urine and FF samples among nonsmokers. Similar results were seen when calculating Pearson correlation coefficients using cotinine concentrations transformed by the natural logarithm (results not shown).

To determine the extent of exposure misclassification based on categories of urinary cotinine as a biomarker of STS exposure, we calculated its percent agreement with FF cotinine categories (Table 3). Agreement between cotinine in urine and FF was poor, and results were consistent among various data stratification approaches (e.g. when using different exposure cutpoints or $\mathrm{Cr}$-adjusted urine). Sensitivity and positive predictive values did strengthen when we classified exposure by a published cutpoint versus the tertile cutpoint, but specificity and negative predictive values decreased proportionally, negating any overall improvement in percent agreement. Finally, urine and FF samples collected closer together in time did not have better agreement than those collected farther apart.

To assess the reliability of FF cotinine concentrations, we calculated intraclass correlation coefficients (ICC) and their 95\% confidence intervals (Table 4). Good reproducibility (ICC $=0.67$ ) was demonstrated when including all participants; though, as expected, when active smokers were excluded, coefficients were reduced for self-reported nonsmokers (ICC $=$ 0.52 ) and "true" nonsmokers (ICC $=0.42$ ). We also assessed the ability of a single FF cotinine measure to correctly categorize women as exposed to STS when compared to their exposure categorization that considered multiple FF cotinine measures. Among 97 women undergoing three or more cycles, FF cotinine agreement was good (Table 5). Results were consistent when active smokers were omitted and when exposure status was defined by a published cutpoint versus self-report.

Finally, since the parent study spanned 10 years we hypothesized that there would be a decline in FF cotinine concentrations over time due to increased tobacco awareness and regulation in the US. However, no temporal trend was observed (results not shown).

\section{DISCUSSION}

In this study, we compared cotinine concentrations in FF and urine samples and examined the temporal variability of FF cotinine measures. Among nonsmoking IVF patients, we found a weak relationship between cotinine levels in paired FF and urine samples. FF cotinine was a more temporally relevant biomarker in the present study because FF samples were collected during ART treatment, while urine samples were often taken before treatment. Since FF is also likely a more biologically relevant sample matrix when assessing STS exposure's effects on early female reproduction, our results provide evidence that measuring cotinine in FF may be a more appropriate biomarker of low-level tobacco smoke exposure in studies of fertility and early pregnancy.

Cotinine in FF and serum correlated highly with one another $\left(\mathrm{R}^{2}=0.95\right.$ and $\left.\mathrm{R}^{2}=0.89\right)$ in two previous reports (Fuentes et al., 2010; Paszkowski, 1998). This inter-fluid cotinine agreement was much stronger than what we observed in the present study, though active smokers were included in both previous studies, likely strengthening the observed correlations. As far as we are aware, only one other study has examined inter-fluid cotinine agreement exclusively among nonsmokers. After dosing subjects with nicotine levels 
intended to simulate STS exposure, Benowitz et al. (2009b) recently reported a strong relationship $\left(\mathrm{R}^{2}=0.84\right)$ between cotinine in blood plasma and Cr-adjusted urine. This reported correlation is also much stronger than what was observed in the present study, but it is difficult to compare results from this study and ours since the relationship between cotinine in blood plasma and FF among nonsmokers is unknown. Paszkowski et al. (1998) found no significant gradient between cotinine in serum and FF, indicating that cotinine easily crosses the blood-follicle barrier; however, $22 \%$ of the subjects in that study were self-reported active smokers. Thus, this finding may be influenced by cotinine-saturated biological compartments within those who actively smoke.

To the best of our knowledge, the present study is also the first to examine intra-individual variability in FF cotinine concentrations. We found moderate to high within-subject variability in FF cotinine concentrations when assessing concentrations as a continuous variable. Our ICC analyses showed fair to poor reproducibility in FF cotinine levels over time, demonstrating the need for multiple FF samples to accurately predict long-term STS exposure. On the other hand, when using FF cotinine concentrations to categorize women as exposed or unexposed, we found a high level of agreement in sensitivity analyses. Thus, if broad exposure categories are used as opposed to continuous FF cotinine concentrations, there may be a smaller degree of exposure misclassification when using a single FF sample to estimate exposure over a longer period of time. However, in our analysis sensitivity may have been overestimated because we used subjects' geometric mean cotinine concentrations as their "gold standard" exposure measure. In other words, the predicted measurements, cotinine measures from a single FF sample, are not independent of the standard against which they are being compared. The lack of independence between these two variables may have increased the observed levels of agreement.

While somewhat low, the ICCs are higher here than what has been seen for other nonpersistent chemicals such as phthalates and bisphenol-A among other studies of infertile couples (Hauser et al., 2004; Mahalingaiah et al., 2008). This may indicate that people are more consistently and repeatedly exposed to STS compared to these other contaminants, perhaps because exposure to the other ubiquitous contaminants is likely to be more multisource, multi-route, and multi-pathway compared to STS exposure.

The variability of cotinine concentrations over time has not been extensively studied in any sample media. In a study examining temporal patterns in urinary cotinine among children whose parents smoked, Matt et al. (2007) reported that over a 7-13 month period, withinsubject variability of urinary cotinine levels was 10-20 times higher than would be expected based on measurement error alone. They also reported that a single urine sample yielded highly accurate estimates of recent exposure only (i.e. 2-3 days) and up to 12 urine samples may be needed to obtain similar precision in estimates of STS exposure over a 4-13 month period. In their study, ICCs for cotinine measured in single urine samples collected four days apart ranged from 0.81 and 0.93 . For cotinine measured in samples collected 138 days or more apart, ICCs ranged from 0.65 to 0.71 . Similarly, in the present study, our subjects' FF samples were collected from one month to a few years apart. We found ICCs of 0.52 and 0.42 for cotinine measured in single FF samples among self-reported nonsmokers and "true" nonsmokers, respectively. We expected ICCs from the present study to be lower than what Matt et al. reported, since all subjects in that study were children whose parents smoked. Matt et al. also reported that ICCs for averaged cotinine measures from multiple samples (between 6 and 15) were much higher than for single samples, at times exceeding 0.93 for time intervals of 138 to 403 days. These findings demonstrate that within-subject cotinine variability increases with time. In other words, a single measure's accuracy as an estimate of exposure decreases as time increase. Thus, collecting and conducting cotinine analysis on 
multiple biological samples over time should improve long-term STS exposure estimate accuracy.

Findings from Matt et al. may represent true variability in exposure to STS over time. However, high between-subject variation in FF cotinine within smoking groups and within women reporting smoking the same number of cigarettes has also been demonstrated (Zenzes et al., 1996), potentially suggesting variation in toxicant metabolism, distribution, and excretion between individuals due to genetic differences (Swan et al., 2005). If so, using a more biologically-relevant exposure biomarker, such as FF, may be vital for minimizing exposure measurement error in epidemiological studies. Alternatively, the results of Zenzes et al. (1996) may serve as further evidence of the inaccuracy of self-reported exposure since self-report was used to create smoking groups in that study.

There are several potential alternate explanations for our findings. Some of the cotinine disagreement we observed between matrices may be the result of using different analytical methods to measure cotinine in urine and FF. However, we expect that this would only account for a small proportion of the observed disagreement because the precision for both methods was good and our sample size was large. Since cotinine levels generally reflect exposure to tobacco smoke within the past 3-4 days (Benowitz et al., 2009a), the time between urine and FF sample collections could also partially explain this lack of agreement. We stratified our correlation and percent agreement analyses, however, by the time between sample collections. In doing so we did not observe an improvement in percent agreement or correlation between samples collected within three days of each other verses all samples. Additionally, relying solely on self-report can lead to exposure misclassification. Thus, for comparison, we used an objective cotinine cutpoint or threshold to determine exposure status. Our results did not change when exposure classification was based upon cutpoints. The use of a first-morning void urine sample may also partially explain the observed disagreement. Other markers of exposure, such as cotinine measured in a 24-hour urine sample, may have been helpful. Unfortunately, such markers were not available in the present study.

Another explanation for our findings may be the difference in storage times of the urine and FF samples. FF samples were frozen/stored for much longer than urine samples before cotinine measurement. The stability of cotinine in FF has not been tested, though the results of a study examining the stability of urinary cotinine and creatinine concentrations suggest that these analytes retain their ability to discriminate between smokers and nonsmokers for at least 10 years of storage at -20 degrees centigrade (Riboli et al., 1995). Also, our findings of weaker within-subject cotinine relationships between FF and urine than between repeat measures from FF collected over time argue that the lack of correlation between urine and FF is true and that cotinine degradation from long-term storage of FF did not occur.

\section{CONCLUSION}

Our results indicate that FF cotinine may be an improved biomarker of exposure to STS compared to urinary cotinine in studies of early pregnancy and that cotinine measures from multiple FF samples may be necessary to accurately represent long-term STS exposure. In the future, we plan to measure cotinine in multiple FF samples from a larger number of women to reevaluate relationships between STS exposure and early pregnancy outcomes.

\section{Acknowledgments}

This work was supported by the Flight Attendant Medical Research Institute (FAMRI) and the NIH National Institute for Environmental Health Sciences (R01 ES013967) and the Eunice Kennedy Shriver National Institute of 
Child Health and Development (R01 HD32153). The authors would like to thank Dr. Craig Harris and Dr. Rita Loch-Caruso for their insights related to the literature review.

\section{References}

Armstrong BG. Effect of measurement error on epidemiological studies of environmental and occupational exposures. Occup Environ Med. 1998; 55:651. [PubMed: 9930084]

Benowitz NL, Hukkanen J, Jacob P. Nicotine chemistry, metabolism, kinetics and biomarkers. Handb Exp Pharmacol. 2009a:29.

Benowitz N, Dains K, Dempsey D, Herrera B, Yu L, Jacob P. Urine nicotine metabolite concentrations in relation to plasma cotinine during low-level nicotine exposure. Nicotine Tob Res. 2009b; 11:954. [PubMed: 19525206]

Boeniger MF, Lowry LK, Rosenberg J. Interpretation of urine results used to assess chemical exposure with emphasis on creatinine adjustments: a review. Am Ind Hyg Assoc J. 1993; 54:615. [PubMed: 8237794]

Cooper AR, Moley KH. Maternal tobacco use and its preimplantation effects on fertility: more reasons to stop smoking. Semin Reprod Med. 2008; 26:204. [Online]. [PubMed: 18302112]

Fabro S. Penetration of chemicals into the oocyte, uterine fluid and preimplantation blastocyst. Environ Health Perspect. 1978; 24:25. [PubMed: 17539150]

Fahiminiya S, Gerard N. Follicular fluid in mammals. Gynecol Obstet Fertil. 2010; 38:402. [Online]. [PubMed: 20576551]

Fortune JE, Rivera GM, Yang MY. Follicular development: the role of the follicular microenvironment in selection of the dominant follicle. Anim Reprod Sci. 2004; 82-83:109.

Fortune JE. Ovarian follicular growth and development in mammals. Biol Reprod. 1994; 50:225. [PubMed: 8142540]

Fuentes A, Muoz A, Barnhart K, Argello B, Daz M, Pommer R. Recent cigarette smoking and assisted reproductive technologies outcome. Fertil Steril. 2010; 93:89. [PubMed: 18973890]

George L, Granath F, Johansson ALV, Annerén G, Cnattingius S. Environmental tobacco smoke and risk of spontaneous abortion. Epidemiology. 2006; 17:500. [PubMed: 16837826]

Hankinson SE, Manson JE, Spiegelman D, Willett WC, Longcope C, Speizer FE. Reproducibility of plasma hormone levels in postmenopausal women over a 2-3-year period. Cancer Epidemiol Biomarkers Prev. 1995; 4:649. [PubMed: 8547832]

Hauser R, Meeker J, Park S, Silva M, Calafat A. Temporal variability of urinary phthalate metabolite levels in men of reproductive age. Environ Health Perspect. 2004; 112:1734. [PubMed: 15579421]

Langone JJ, Gjika HB, Van Vunakis H. Nicotine and its metabolites: radioimmunoassays for nicotine and cotinine. Biochemistry. 1973; 12:5025. [Online]. [PubMed: 4761980]

Mahalingaiah S, Meeker J, Pearson K, Calafat A, Ye X, Petrozza J, Hauser R. Temporal variability and predictors of urinary bisphenol A concentrations in men and women. Environ Health Perspect. 2008; 116:173. [PubMed: 18288314]

Matt GE, Hovell MF, Quintana PJE, Zakarian J, Liles S, Meltzer SB, Benowitz NL. The variability of urinary cotinine levels in young children: implications for measuring ETS exposure. Nicotine Tob Res. 2007; 9:83. [PubMed: 17365739]

Meeker JD, Missmer SA, Cramer DW, Hauser R. Maternal exposure to secondhand tobacco smoke and pregnancy outcome among couples undergoing assisted reproduction. Hum Reprod. 2007; 22:337. [Online]. [PubMed: 17053002]

Meeker J, Barr D, Ryan L, Herrick R, Bennett D, Bravo R, Hauser R. Temporal variability of urinary levels of nonpersistent insecticides in adult men. J Expo Anal Environ Epidemiol. 2005; 15:271. [PubMed: 15340359]

Neal M, Hughes E, Holloway A, Foster W. Sidestream smoking is equally as damaging as mainstream smoking on IVF outcomes. Hum Reprod. 2005; 20:2531. [Online]. [PubMed: 15919779]

Paszkowski T. Concentration gradient of cotinine between blood serum and preovulatory follicular fluid. Ginekol Pol. 1998; 69:1131. [PubMed: 10224789]

Peppone LJ, Piazza KM, Mahoney MC, Morrow GR, Mustian KM, Palesh OG, Hyland A. Associations between adult and childhood secondhand smoke exposures and fecundity and fetal 
loss among women who visited a cancer hospital. Tob Control. 2009; 18:115. [PubMed: 19039010]

Riboli E, Haley N, De Waard F, Saracci R. Validity of urinary biomarkers of exposure to tobacco smoke following prolonged storage. Int J Epidemiol. 1995; 24:354. [PubMed: 7635596]

Rosner, B. Fundamentals of Biostatistics. 5. Duxbury; Pacific Grove, CA: 2000.

Sterzik K, Strehler E, De Santo M, Trumpp N, Abt M, Rosenbusch B, Schneider A. Influence of smoking on fertility in women attending an in vitro fertilization program. Fertil Steril. 1996; 65:810. [PubMed: 8654644]

Swan GE, Benowitz NL, Lessov CN, Jacob P, Tyndale RF, Wilhelmsen K. Nicotine metabolism: the impact of CYP2A6 on estimates of additive genetic influence. Pharmacogenet Genomics. 2005; 15:115. [PubMed: 15861035]

Van Vunakis, H.; Gijka, HB.; Langone, JJ. Radioimmunoassay for Nicotine and Cotinine. International Agency for Research on Cancer; Lyon, France: 1993.

Weber PA, Chang HC, Spaeth KE, Nitsche JM, Nicholson BJ. The permeability of gap junction channels to probes of different size is dependent on connexin composition and permeant-pore affinities. Biophys J. 2004; 87:958. [PubMed: 15298902]

Weiss T, Eckert A. Cotinine levels in follicular fluid and serum of IVF patients: effect on granulosaluteal cell function in vitro. Hum Reprod. 1989; 4:482. [PubMed: 2794009]

Zenzes MT, Reed TE. Interovarian differences in levels of cotinine, a major metabolite of nicotine, in women undergoing IVF who are exposed to cigarette smoke. J Assist Reprod Genet. 1998; 15:99. [PubMed: 9513850]

Zenzes MT, Reed TE, Wang P, Klein J. Cotinine, a major metabolite of nicotine, is detectable in follicular fluids of passive smokers in in vitro fertilization therapy. Fertil Steril. 1996; 66:614. [Online]. [PubMed: 8816626]

Zielińska-Danch W, Wardas W, Sobczak A, Szołtysek-Bołdys I. Estimation of urinary cotinine cut-off points distinguishing non-smokers, passive and active smokers. Biomarkers. 2007; 12:484.

[PubMed: 17701747] 
Figure 1.

Urinary cotinine versus follicular fluid cotinine in paired samples from the same women (N $=242$ self-reported nonsmokers) 


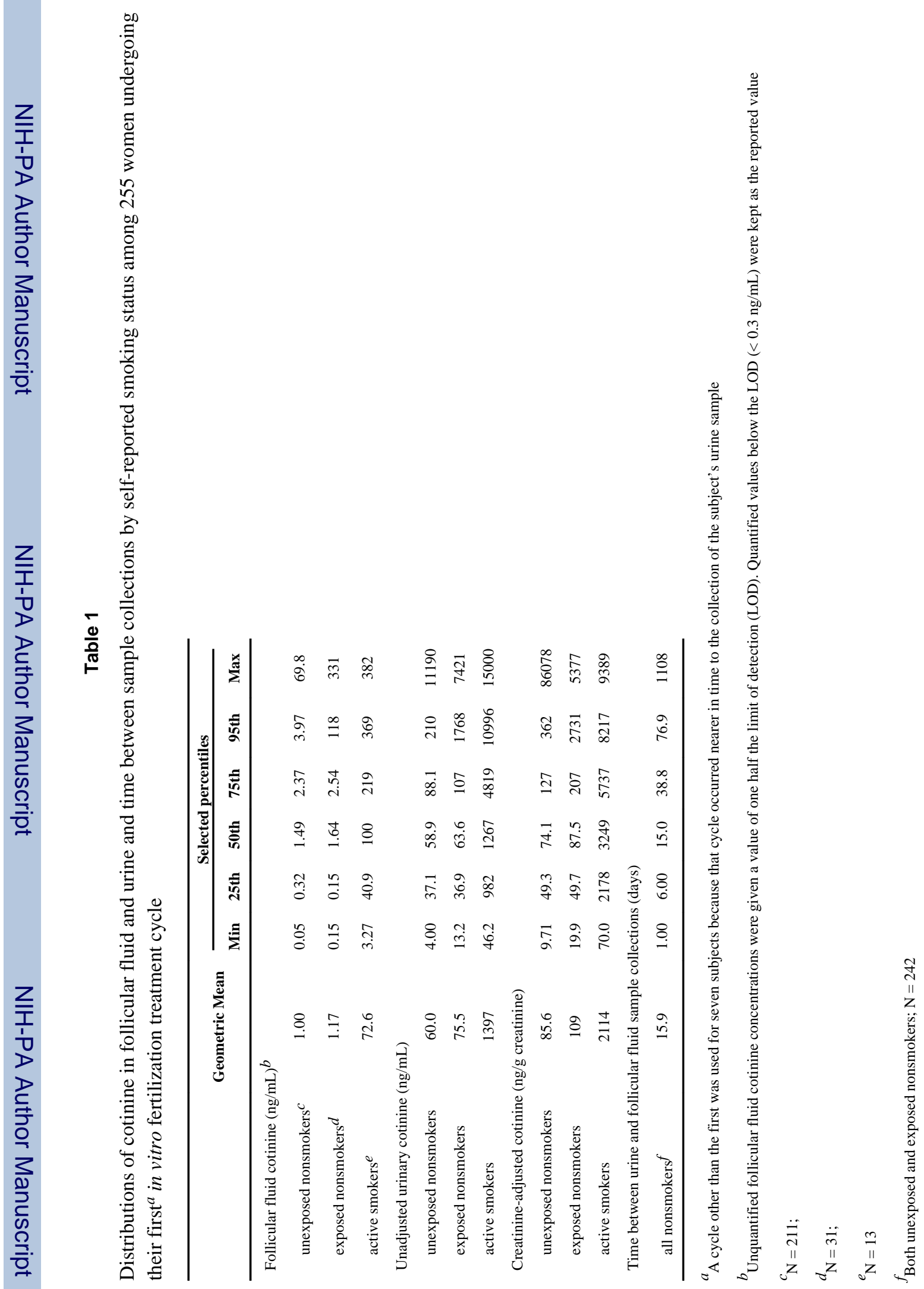


Table 2

Spearman correlation coefficients between cotinine measured in follicular fluid and urine samples among nonsmoking in vitro fertilization patients

\begin{tabular}{|c|c|c|c|}
\hline & $\mathbf{N}$ & Unadjusted urine & Creatinine-adjusted urine \\
\hline \multicolumn{4}{|c|}{ Nonsmokers determined by self-report } \\
\hline all samples & 242 & $0.13^{a}$ & $0.15^{a}$ \\
\hline$>15$ days apart & 122 & 0.06 & 0.13 \\
\hline$\leq 15$ days apart & 120 & $0.20^{a}$ & 0.16 \\
\hline$\leq 7$ days apart & 82 & 0.14 & 0.11 \\
\hline$\leq 3$ days apart & 17 & 0.12 & -0.08 \\
\hline \multicolumn{4}{|c|}{ Nonsmokers determined by published cutoff $b$} \\
\hline all samples & 238 & 0.07 & 0.08 \\
\hline$>15$ days apart & 120 & 0.01 & 0.08 \\
\hline$\leq 15$ days apart & 118 & 0.12 & 0.07 \\
\hline$\leq 7$ days apart & 80 & 0.08 & 0.04 \\
\hline$\leq 3$ days apart & 16 & -0.06 & -0.29 \\
\hline $\mathrm{p}$-value $\leq 0.05$ & & & \\
\hline
\end{tabular}




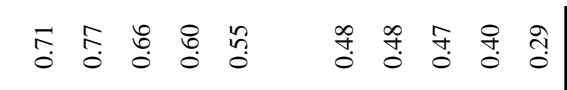

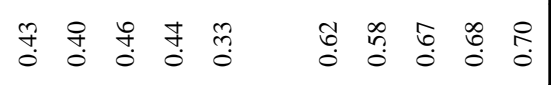

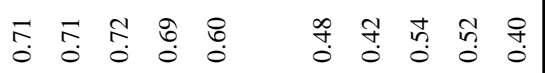

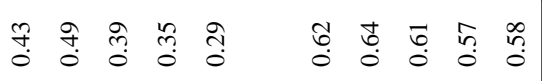

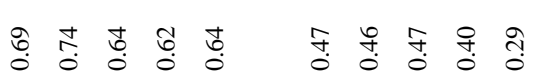

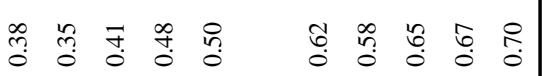

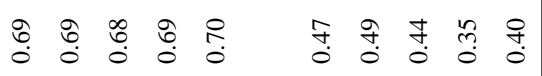

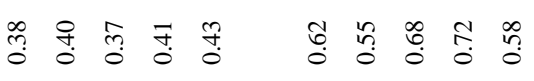

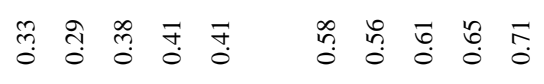

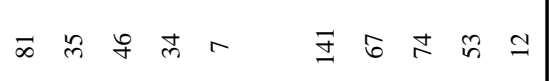

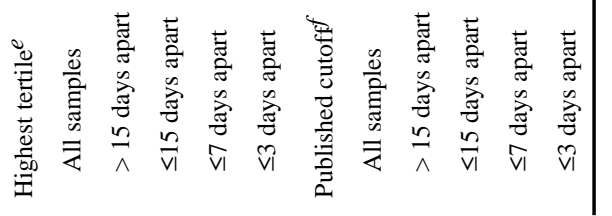

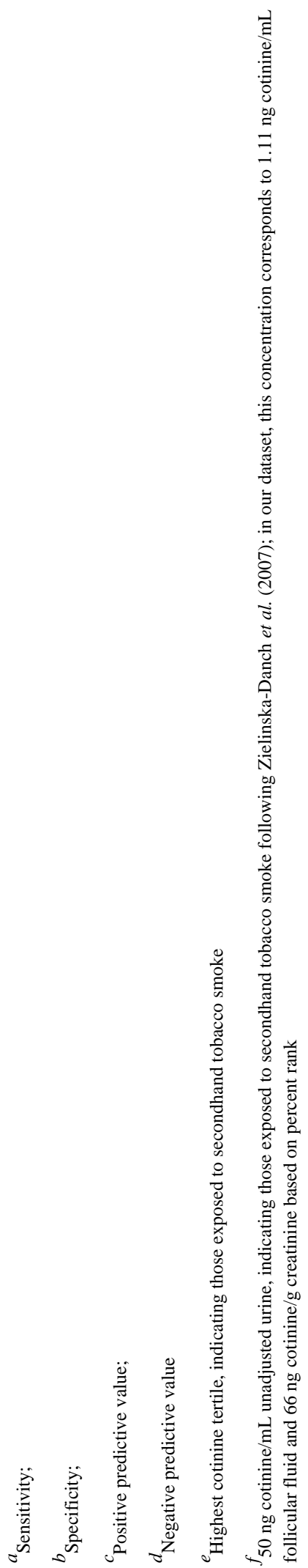




\section{Table 4}

Intraclass correlation coefficients (ICC) for cotinine levels in repeated follicular fluid samples

\begin{tabular}{lcccc}
\hline & No. of women & No. of cycles & ICC & 95\% CI \\
\hline All subjects & 415 & 765 & 0.67 & $0.61-0.73$ \\
Self-reported nonsmokers & 388 & 718 & 0.52 & $0.44-0.59$ \\
"True" nonsmokers $a$ & 379 & 696 & 0.42 & $0.34-0.52$ \\
\hline
\end{tabular}

${ }^{a}$ Geometric mean below $10 \mathrm{ng}$ cotinine/mL follicular fluid, indicating participants who are not active smokers following Fuentes et al. (2010) 


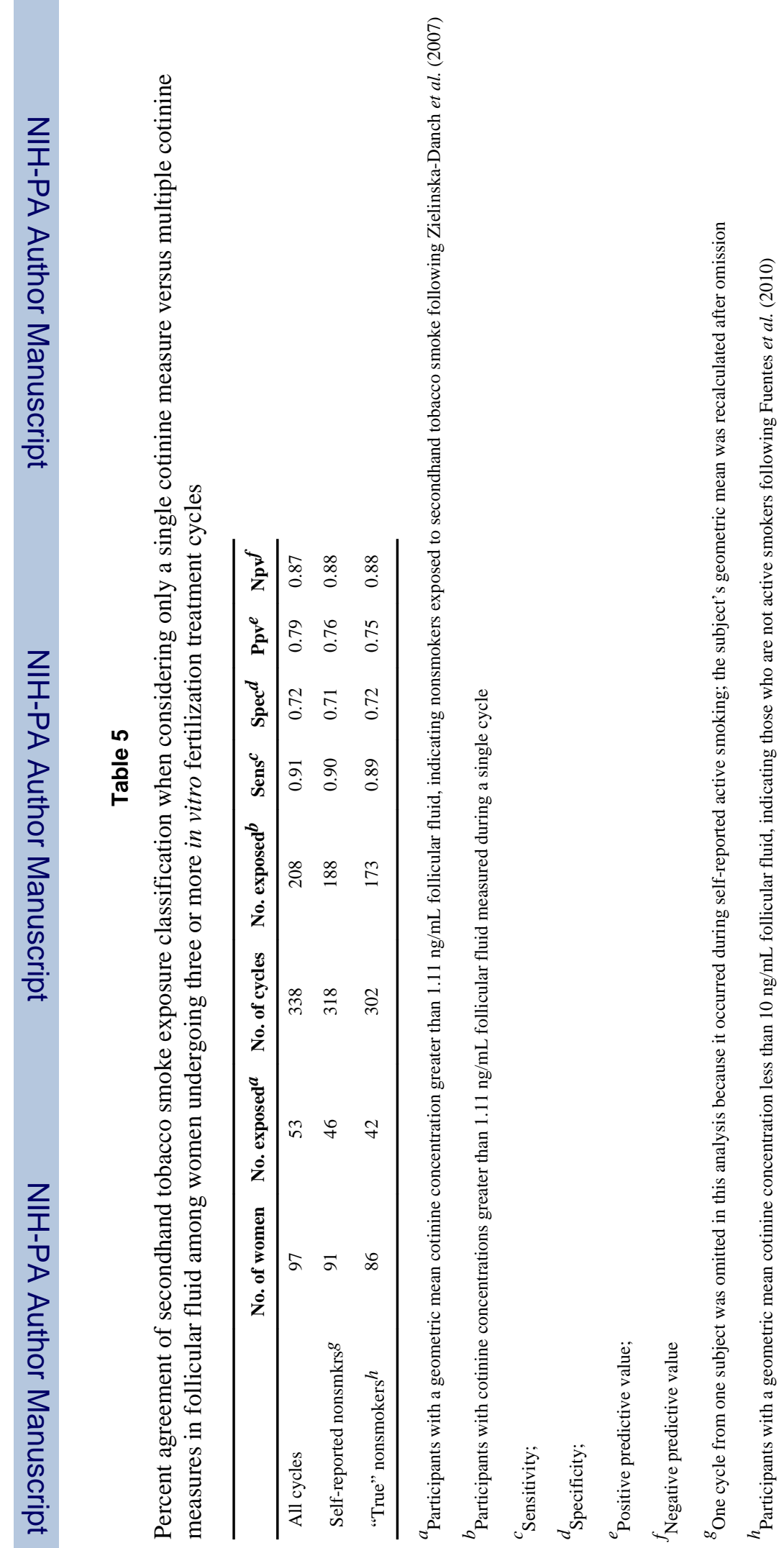

Chemosphere. Author manuscript; available in PMC 2012 June 1. 